\title{
Examining the Reuse of Qualitative Research Data: Digital Video in Education
}

\author{
Rebecca D. Frank, Kara Suzuka, Elizabeth Yakel
}

\begin{abstract}
Qualitative data, particularly digital video, present unique challenges for reuse. These challenges include the need for contextual data, privacy and confidentiality concerns, and technical issues involving search, data manipulation, analysis, and capture of selected scenes for teaching. In this article we examine the reuse practices of users from an educational repository and focus on the latter challenge - the ways that tools for discovery, access, and use influence data reuse among researchers, teacher-educators, and teachers in practice. We identify major issues such as reusers' difficulties with video data management, the challenge of research collaborations with digital video, and preferences for common and familiar tools over specialized software to remix and/or analyze the video.
\end{abstract}

\section{Suggested Citation}

Frank, R. D., Suzuka, K., \& Yakel, E. (2016). Examining the Reuse of Qualitative Research Data: Digital Video in Education. In Archiving Conference (Vol. 2016, pp. 146-151). Washington, DC: Society for Imaging Science and Technology. https://doi.org/10.2352/issn.2168-3204.2016.1.0.146

\section{Copyright Notice}

"Permission to make digital or hard copies of part or all of this work for personal or classroom use is granted without fee provided that copies are not made or distributed for profit or commercial advantage and that copies bear this notice and the full citation on the first page. Copyrights for components of this work owned by others than IS\&T must be honored. Abstracting with credit is permitted. To copy otherwise, to republish, to post on servers or to redistribute to lists, requires prior specific permission and/or a fee."

[Final publication is available at:

http://www.ingentaconnect.com/content/ist/ac/2016/00002016/00000001/art00032]

\section{Rebecca D. Frank}

School of Information, University of Michigan, 3429 North Quad, 105 S. State St., Ann Arbor, MI 48109-1285; frankrd@umich.edu

\section{Kara Suzuka}

School of Education, University of Michigan, 610 E. University Ave., Ann Arbor, Michigan 48109-1259; ksuzuka@umich.edu

\section{Elizabeth Yakel}

School of Information, University of Michigan, 4323 North Quad, 105 S. State St., Ann Arbor, MI 48109-1285; yakel@umich.edu 


\section{Introduction}

Data reuse depends on the ability of users to discover, access, and work with data. While recent research has explored the reuse of quantitative data extensively, far less attention has been given to the reuse of qualitative data [1]-[3]. This article reports on the results of a qualitative study that focuses specifically on the reuse of qualitative data, particularly the reuse of digital videos and other digital records of practice, by educational researchers, teacher-educators, and teachers themselves.

In education, digital records of practice are "detailed documentation of teaching and learning...taken directly from teaching and learning, without analysis, which enable (people) to look at practice" [1]. These records include video of teachers and students in situ, working on learning objectives or classroom activities. These are also naturally occurring records, such as teachers' lesson plans or student assignments; as well as contextual data such as seating charts, demographic information about students (e.g., English as a second language status) or the school district (e.g., number of federally supported breakfasts or lunches). We investigate the ways that the reuse of digital records of practice are influenced by the tools that repositories employ or users select, and argue that effective reuse of qualitative video data is influenced by tool selection.

\section{Motivation/Literature}

As data reuse increases and repositories seek to meet the needs of a wide array of users, there is a need for research that identifies and addresses the unique challenges that qualitative data presents for repositories and users [4]-[7]. The field of education has a long history of using video records of practice to study learning and teaching in research and educational efforts [8]-[10] as a way to explore the complex interactions and cognitive activities that take place in a classroom environment [8], [11].

However, digital records of practice that capture classroom activities present unique challenges for reuse. These videos often capture many individuals (e.g. in-service teachers, pre-service teachers, students) and complex processes, such as comprehension and learning, communication (e.g. instruction), presenting "social, ethical, legal, and institutional" obstacles for reuse [12, p. 636]. Repositories that preserve and provide access to these digital records of practice must address both the technical challenges of managing digital video [13]-[15], and also social challenges, such as understanding learning objectives [4].

In this article, we examine the qualitative data reuse practices of repository users in order to understand the technological challenges, specifically the tools that they use and the problems experienced when accessing, understanding, and using those tools. We focus on users in the field of education for two reasons. First, the field has a long history of using qualitative data, particularly video data [8], [16]. Second, the designated community of education presents three different types of data reusers with distinct needs: researchers, teacher-educators who use the video in pre-service teacher-education and in professional development for practicing teachers, and finally teachers who use the videos for personal study.

\section{Problem}

Our study is motivated by two research questions:

- What tools do researchers, teacher-educators, and teachers use to locate, access, and use/reuse educational digital records of practice?

- How does tool selection influence the ways researchers, teacher-educators, and teachers use/reuse digital records of practice? 


\section{Methods}

We conducted a qualitative study consisting of ten in-depth semi-structured interviews with users of multiple repositories containing records of practice relating to learning and teaching. In the interviews, we asked how respondents located and used digital records of practice, the specific tools that they used to work with digital video, and the problems that they encountered. The tools described by respondents included video/audio/image editing software (e.g., iMovie, Final Cut), video commenting software/platforms (e.g., Vimeo, Edthena), video data analysis software (e.g., Transana, NVivo), video authoring software/platforms (e.g., Zaption, Adobe Captivate), video sharing software/platforms, and presentation software (e.g. PowerPoint, Keynote). The interview transcripts were analyzed with a code set based on concepts from the literature as well as themes that emerged during the coding process. The set included codes about tool identification, tool selection, tool use, and challenges.

The interviewees consisted of 6 higher education faculty members, 1 graduate student, 1 education policy administrator, and 2 primary/secondary school faculty members. The higher education faculty members and graduate students reported using digital records of practice for research and teacher education, while the education policy administrator and primary/secondary school faculty members used digital records of practice for personal study and teaching. For all users, collaboration with others was an important aspect of their use and reuse of digital records of practice.

\section{Results}

Users' ability to access and work with digital records of practice was both enabled and limited by their knowledge of data available for reuse and their level of comfort with the tools that were available. The interviews demonstrated that while users were aware of some repositories curating digital records of practice, they were not aware of the entire universe of data. In terms of tools, preliminary results show that most users made limited use of digital tools to capture, manage, analyze, and present data. Underutilization of both data and tools was a major barrier to expanding data reuse.

In this section, we begin with a brief description of researchers', teacher-educators', and teachers work practices around digital video. We then examine the interaction of work practice and tools on two core activities: video data management by teacher-educators and collaborative research. We end with a discussion of tool selection and preferences for common and familiar tools over specialized software to remix and/or analyze the video by all types of video data reusers.

\section{Work Practices Using Educational Digital Records of Practice}

Participants reported using a wide variety of tools to access and work with educational digital records of practice. Some tools were repository-based, and others were selected and brought to the video by the users or their collaborators. Study participants described their use of digital records of practice in three primary areas: research; classroom instruction and teacher education; and personal study. Digital video serves a critical function in all of these areas. Using digital records of practice for research involved finding and accessing, analyzing, storing, and sharing data. This is a highly collaborative process as few individual researchers can capture and analyze sufficient video data to generate strong findings. Therefore collaborative data capture, data sharing, and collaborative analysis and publication are the norm. In some cases, decisions about which data to use were driven by availability and convenience, and in others selection was purpose-driven regardless of the difficulty in accessing the data. Interaction with tools is prevalent in all stages of the research process: finding and accessing data, data analysis, data sharing, and reporting on or with digital records of practice.

For teacher-educators and teachers engaged in personal study, video provided a window on others' teaching practices; new ideas and methods for communicating concepts particularly in math and science; and an opportunity to reflect on one's own teaching. Finding the 'right' video clip was key. These users were likely to 
then reuse that clip frequently rather than re-engage in a search for alternatives. Teachers engaged in personal study are also purpose-driven, looking for a video demonstrating instruction of a particular concept or topic for inspiration and/or replication.

For teacher-educators and teachers engaged in personal study, being able to easily locate relevant video was key. QDR_009 argued that poor organization and discoverability of digital records of practice were partly to blame for low levels of adoption of video in instruction by practicing teachers for professional development, "it seems to me that if the libraries of videos were out there well-organized, searchable, done in whatever the right way would be, that professional development at the school level and at the district level could rely much more heavily on videos and really promote that kind of... Promote discussion in teams or on faculties of certain things that people may be wanting to focus on." For some users, lack of usable tools for discovery, or tools that present obstacles to discovery, result in a failure to effectively use a repository. Educational repositories have petabytes of digital video data but the search tools have not caught up with that, and users are either unable or unwilling to spend the time that it would take to wade through the high volume of information in order to find what amounts to a needle in a haystack.

Many of these work practices require that users of digital records of practice manage large amounts of data. Whether they are responsible for data storage or not, use of these records requires the ability to manage and navigate collections of digital video and other digital artifacts that cannot be easily or quickly browsed. The ways in which users respond to these data management challenges, or fail to respond to them, both enable and limit their ability to utilize digital records of practice.

\section{Managing Data}

For all three user groups, managing personal collections of videos was challenging. Participants voiced concerns about managing digital records of practice, especially with regard to their own personal collections of data. Because many reusers preferred to download digital records of practice for their own use, they ended up having to manage large and often unwieldy amounts of data within their own personal collections. Both QDR_001 and QDR_006 described having difficulty managing their own files, and specifically discussed problems locating (and re-locating) specific videos, whether those files were located on their own hard drives or elsewhere, “And so just getting access to the videos, and there's such a big corpus of material right now. And so frequently I want to look at something and I sort of forget where things were stored or the places I thought I have them, they no longer function" (QDR_001).

For some, naming and organizing files, and locating and identifying videos or clips, proved most difficult, "So, for a while I was labeling things "video" in the title, and I was just like... But I can look at the icon next to it and see that it's video, so why am I doing that? That doesn't necessarily make any sense. But there were pieces of information that were really important about that data that weren't getting surfaced, and so then I'd have to go back and look at 120 files to find it to rename it in a way that was helpful later" (QDR_002).

Likewise, QDR_006 also described having trouble managing her own files, giving the example of poorly named files that did not provide sufficient information to help her choose particular clips, "Well, that's sort of one of the things I need to do. Right now, they're all in a folder. And when I need to do is... What I wanna do is just sort of organize them. They are somewhat organized by the source. Like I have a folder of classroom discussions clip. But what's stupid is, I don't have them named in any way, other than I... So, I click on them and I see which one it is, it's sort of ridiculous. I mean, they all have a name, but the name doesn't mean anything to me. My clips are all just sort of in there and I look up again, "Oh, which one is that? Oh, yeah. That's 1A, I wanna show that one." It's very poorly organized. Or, it's like in another PowerPoint, and I just clip..." (QDR_006). Having more files than could be easily managed made it difficult for this participant to search through her personal library in order to find specific records of practice to meet her needs. 
QDR_010 described challenges in managing a large personal library of digital video but also expressed hope that learning to use the tool StudioCode, a video analysis software that enables coding and annotation of digital video, would result in better data management in the future, "I'm just starting to get into using StudioCode, learning how to use StudioCode to do my own tagging and analysis of our professional development videos. But moving forward, I think as I have a larger kind of library of coded videos in StudioCode, those will be easier to mine for clips that I wanna use. All my videos are in QuickTime, and so I'm constantly clipping and saving stuff in QuickTime. One thing that I find I need help with is just keeping track of everything, right? The reason that I know that I have it, so I just finished teaching a course, and the reason I picked the video to use on a particular day was because that's what I had used the year before, right? If I've looked at my whole corpus of data, there probably is a better video to use, but this is the one I have so this is the one I'm using. Or this is the one I'm using because this is the one I'm using" (QDR_010).

All activities where video files were stored and organized on a user's local storage depended upon personal, idiosyncratic, and ad hoc organization for storing and, more critically, being able to search and discover these files when they were wanted or needed again at some point in the future. This problem was made even more complicated when collaborating with others who had their own idiosyncratic systems. Problems managing data became more complex when users were collaborating with others. It was infeasible to rely on personal memory of particular lectures or events to locate videos or clips, as collaborators did not have the same level of knowledge about the collection of digital records of practice. Tools that support data management might help teams manage data, but these also present some barriers to collaboration.

\section{Collaboration}

Collaboration with others drove tool selection in both positive and negative ways. Participants reported selecting particular tools that enabled collaboration for both research and teaching with digital records of practice, but they also noted that collaboration limited their choice of tools.

QDR_005 described using one particular platform, Edthena, because of the tools available via that platform for annotating and commenting in digital videos. These tools enabled collaboration with pre-service teacher education students and instructors by allowing them to easily share and comment on video together without needing to synchronize multiple artifacts or files, "For my work as a teacher educator, Edthena is quite crucial. Just because of the ability to be able to give comments on specific moments in the teaching, and to make it really easy for someone else to go back into hit that point and to be able to look at it" (QDR_005).

In some cases, however, the choice of tools for annotating and commenting on the digital video depended on collaborators' previous tool selections. For example, QDR_005's tool choices differed when working alone than in collaborations. "I use Edthena quite a bit in the context of my own teaching, and I don't know, I'm trying to think if I have ever used it when working with colleagues on video. I don't think I have used it as much when working with colleagues on video just in part because of not all of my colleagues have access to Edthena" (QDR_005). In this case, she chose not to use Edthena when working with colleagues because some did not have access (Edthena is a paid service). Limitations on access to tools for working with data served to restrict tool use even when a specific tool might be the best option for a particular task.

Similarly, QDR_010 discussed several factors that contributed to tool selection, highlighting the need for software and services to enable sharing with collaborators and colleagues, as well as the importance of convenience in making videos discoverable. "Dropbox, yeah. [Collaborator] and I share videos in Dropbox." (QDR_010).

An approach relying on multiple software and additional documents was also described in relation to collaborative research. QDR_004 described a project in which team members were able to watch digital video 
with any video playing tool that allowed collaborators to easily use "markers" inserted into the video - something that would allow everyone to play and replay the same segments efficiently. The coding and analysis of data then took place in an Excel spreadsheet, "The way we've been coding we've been getting some sort of tool or getting the video onto a platform or where things can be replayed easily, where markers are easy to install. For instance, we watched a video in five-minute clips, and coded on the five minutes. So, being able to quickly maneuver so that you can play five minutes and then go back and replay that five minutes if you want or not or... That kind of thing, but most video viewing software is fine for that" (QDR_004). For this participant, it was important to devise a system that would allow all members of the research team to easily view pre-specified five-minute segments of digital video while collaboratively using Excel, a commonly available program, for coding the data. Although this added a level of complexity by generating additional files for the team to manage, separating the video data from the coding made analysis by a team possible, overcoming some of the potential challenges described by other participants above (e.g. QDR_005 and QDR_010).

QDR_002 also characterized tools as a site of struggle among researchers seeking to collaborate. According to her, the amount of work involved in learning a new tool or set of tools to collaborate with different research teams increased the cognitive load on the individuals, potentially taking up time and attention that could be spent on the research itself, "I think, and this might sound silly, but the primary struggle is I feel like everybody is using different tools. Everyone's got a different video software and everyone seems to be using different coding software and everyone seems to be using different... People use things from Word to Excel to actually specialized software to organize those things. It seems overwhelming and every single time I have to - it's a huge cognitive load because every single time I join a project or pick up a project or help or consult on a project I have to learn a new system or set of systems too 'cause there are usually three different things you have to learn, you have to add them all to your computer" (QDR_002).

This participant went on to explain that the prospect of having to learn new tools and systems to work with data prevented her from joining projects and working with other collaborators, "And I really struggle with that to a point where I've actually said no to two projects this year because I had no desire to learn new coding software. I just wouldn't do it. I chose to use Dedoose for my own dissertation 'cause it was the easiest thing in the world to use, not because necessarily it was the most appropriate tool. But at that point I was just like, 'I don't want to have to learn another NVivo. I don't wanna have to learn another Stata. I don't want to have to learn another.' ... That's fatiguing, I think it's a huge problem for me personally" (QDR_002). For this participant, the challenge not only of learning new and potentially complicated tools and programs but also of learning new workflows and processes (some of which rely on relatively simple or commonly used programs) has been a barrier to collaboration. Satisficing with a tool that would get the job done, (i.e. Dedoose) and was already familiar, was preferable to finding a more appropriate tool that she would have to learn.

When streaming video rather than downloading, participants voiced concerns about data confidentiality. In particular, participants whose use of digital video focused on research rather than teaching were concerned about sharing their own data with collaborators or other potential reusers in ways that would meet data confidentiality requirements dictated by their institutional review boards (IRBs). QDR_005 explained, "Yeah. So I've used different things. So for some work with colleagues at other institutions we've been using Vimeo temporarily while we work on our data sharing agreement ... Cause I felt like it was still a place where it was pretty locked down, and I was comfortable with that, but I wasn't gonna hand them a file." For this researcher, security and the ability to limit access to data were important features in tool selection for sharing and viewing digital records of practice. One way to control this access was through the use of a tool that would let collaborators view digital video without being able to download it to their own hard drive.

The problems and challenges described by participants above affected work practices, data management, and collaboration and also influenced tool selection. In particular, we found that respondents tended to choose tools with low barriers to entry for their work with digital records of practice whenever possible. 
Frank, et al: Examining the Reuse of Digital Video as Qualitative Data

\section{Tools with Low Barriers to Entry}

For our interview participants, their choice of tools for finding, accessing, and working with digital records of practice were influenced by their familiarity and comfort with the technology, their knowledge of available tools and programs, their levels of access to potentially expensive tools and services, the challenges of managing large amounts of data, and considerations about their collaborators and students. For many participants, reliable access to the Internet was not guaranteed and so tool selection was also limited by the need to be able to work or teach with the video offline.

Participants described different work environments and tools for research, in many cases creating unique combinations of basic tools (e.g. Microsoft Excel and Google docs) in order to accomplish their work, rather than using specialized, purpose-built tools for working with digital video or other qualitative data (e.g. Transana, NVivo).

QDR_001 described using additional documents to enable analysis of the digital records of practice. Rather than using a program to code or annotate the video directly, he explained that he annotates the lesson plan, adding in timestamps to indicate where in the video particular events occur, "So by annotating it onto the lesson plan, then automatically locates it where. So if you said it happened 23 minutes into the lesson, then you could look for it in the video" (QDR_001). The practice of using accompanying documentation to create a roadmap for the video is reflected in the practices described by several participants, who use programs such as Excel or Google Docs to analyze and code their data rather than programs such as StudioCode, which would allow coding and annotation within the same file as the video. Rather than having to rely on file names, or on watching numerous videos, QDR_001 has been able to use a document to flag particular elements of interest. Using accompanying artifacts as roadmaps to the videos is one way that participants overcame the data location and analysis challenges described by participants such as QDR_006 and QDR_010.

Using digital records of practice for classroom instruction and teacher education also involves using an array of tools to find, access, and work with digital video, but these users focused more on sharing that video in an instructional setting rather than conducting data analysis activities, such as coding. Participants who used digital records of practice in this way tended to select basic tools for viewing and editing video (e.g. QuickTime) in order to show specific clips or segments during instruction. Many also cited workarounds, such as writing down a particular timestamp, as a way to show part of a video without having to learn how to use tools for video editing (e.g. iMovie).

Data reusers' choice of tools for viewing digital video depended in part on whether they had a reliable Internet connection. This was particularly the case or teacher-educators engaged in professional development and teachers using video for personal study. Participants noted that Internet connections in schools were often unreliable and so videos must be available without relying on streaming access, "In general, I put videos in PowerPoint presentations. Just in part, because it's so hard to know in a particular room whether Internet access is going to be what it should be. There's nothing worse than going to hit the play button, and not being able to show your video" (QDR_005). This consideration varied among participants depending on the context of their teaching. "So I, the classroom that I teach in, I can stream videos pretty reliably. So for my online courses and for my courses that I teach on campus, I'll use either videos that I can download and just play from my hard drive, or videos that I stream. When we do professional development in schools, Internet is not as reliable, so if we wanna exemplify something before we go teach it, we usually rely on videos that we can download and play from our hard drive" (QDR_010). For these participants, Internet access determined which types of tools could be used for accessing and working with digital records of practice depending on their location.

Participants most frequently discussed using PowerPoint for sharing digital records of practice in the context of teaching or presentations to groups, "PowerPoint, right? Not very exciting... but then in order to really elaborate our findings - we had three sets of findings - we took a piece of the transcript that we created, and so 
you see (the relevant portion of the transcript animates to appear large and bolded on the PowerPoint slide, standing out from the rest of the transcript). Remember how I said before in the end there's just one row, and that's how it looks like in the end. And then, we wanted to bring out particular points in that transcript"

(QDR_003). Teachers also found that embedding digital records of practice in PowerPoint slides maintained the flow of their lesson, while keeping additional text or information on the screen along with the video, "It's just so, it's a little less clunky than having to pop out and pop back in. I mean, although I do it all the time. It's not that big a deal. But I prefer to have within there and then it's all good. And another reason, I'll tell you is because generally, when I play a video, I have the citation on the screen below. And, if I leave and play it from another source, you can't always tell what the source is. So, I just wanna put the citation of where the video came from" (QDR_006).

One of the challenges associated with using PowerPoint to show digital video was the fact that the user must capture a small clip to embed in the presentation. QDR_001 discussed having difficulty in taking clips of full videos to put into presentations for sharing, "Yeah. I mean, they [barriers to access] exist. So even getting video in editable form where you can cut out sections of the video to put in say, to a PowerPoint presentation or something like that. So the usability of the format, so some of it..." (QDR_001). For QDR_001, if smaller clips from full videos were not available, he made note of the place in the video to show and jumped to that point in the video. He was uncomfortable with video editing software, describing himself as not particularly tech-savvy, "I've looked at quite a few of those videos and used them sometimes. I mean, they're full classes and I don't know, I've never tried to edit pieces of it. Although, it's easy when you know what you want to look at. You can sort of play only a segment of it" (QDR_001).

Participants using digital records of practice for personal study described finding and accessing digital video, and managing their own personal collection of videos. In many cases they shared videos with others, but this was generally through a process of exchanging URLs for publicly available videos rather than transferring files directly from one person to another. These reusers utilized basic tools for viewing digital video, but did not use editing or annotation tools in any way.

\section{Discussion}

These findings suggest several implications for repositories. For example, there are several areas where repositories can offer support to users by focusing on key issues of working with digital records of practice - e.g. supports for data management, interoperable collaboration, and leveraging specialized proprietary and commonly available tools.

Participants above described challenges in finding digital records of practice to meet their needs. Repositorybased tools for search and discovery have not caught up with the vast amounts of video data available to users. This is essentially a metadata problem but only with improvements in this area will users be able to take advantage of the available data and support effective reuse. Supporting discovery and access for reuse is particularly important given how time-consuming and costly data collection can be in this space.

Many participants also preferred downloading digital videos to their own computers for use, in many cases because the contexts in which they tended to use the videos did not always have reliable Internet. Once identified and downloaded, data reusers had problems managing large amounts of digital video. Data resuers, particularly teacher educators and those engaged in personal study, had trouble organizing the video and then re-finding appropriate examples. Personal file systems were used over programs designed to enable more fine-tuned organization and retrieval of video. This often led to continued use of the same clips over and over.

While researchers faced issues managing data, they more frequently raised the issue of tool selection in research collaborations, a necessity in this field of study. Tools could be a major reason for or against collaboration. Collaborators had to negotiate what tools would be used in every stage of the research: sharing 
data, analysis, and visualizing results. Reaching a consensus resulted in different configurations of tools from a one size fits all approach (e.g. everyone on the team using StudioCode) to collaborations where each researcher used multiple tools to view video then shared a common and easy-to-use analysis tool, such as Google Sheets or Excel, to collaboratively carry out the coding.

Across all groups of data reusers, participants demonstrated preferences for using combinations of tools with low barriers to entry, e.g., those that were commonly used for other purposes such as PowerPoint, Excel, Google Docs, and QuickTime, rather than specialized tools. These were not only familiar and had a negligible learning curve, but were often more readily available to all team members involved in the collaboration.

Repositories in this space have tended to focus inwardly and on the management of their own data. But a pivot toward reusers would go a long way. Repositories could assist personal data management by ensuring that the video data and any available metadata are downloaded together, enhancing findability on personal computers - as well as supporting user education of best practices for personal data management. While repositories tend to use more specialized, proprietary tools, thinking about how these interface with more commonly available and familiar tools would enable more effective reuse. Given that there are no industry-leader tools and all repositories use different combinations of these tools, this is an important consideration. Repositories could also investigate how to provide multiple options for working with the data to better support all types of data reusers,

\section{Conclusions}

In this research we have presented a qualitative study examining the reuse of digital records of practice by educational researchers, teacher-educators, and teachers. We found that the ability of users to access and work with qualitative data was facilitated and limited by their knowledge of available data for reuse as well as their comfort and ability levels with the tools that were available. Underutilization of data and tools was a major barrier to expanding data reuse. Repositories can better support the needs of their users by ensuring that data and metadata can be downloaded together to enhance findability on personal computers, and by supporting commonly available and familiar tools in order to enable more effective data reuse.

\section{Acknowledgements}

This research is funded by the Institute of Museum and Library Services \# LG-06-14-0122-14.

\section{References}

[1] Dallmeier-Tiessen, S., Darby, R., Gitmans, K., Lambert, S., Matthews, B., Mele, S., Suhonen, J., and Wilson, M., 2014, "Enabling Sharing and Reuse of Scientific Data," New Review of Information Networking, 19(1), pp. 16-43.

[2] Davidson, J., Jones, S., and Molloy, L., 2014, "Big Data: The Potential Role of Research Data Management and Research Data Registries," Lyon, France.

[3] Hicks, B. J., Culley, S. J., Allen, R. D., and Mullineux, G., 2002, “A framework for the requirements of capturing, storing and reusing information and knowledge in engineering design," International Journal of Information Management, 22(4), pp. 263-280.

[4] Bishop, L., 2009, "Ethical Sharing and Reuse of Qualitative Data,” Australian Journal of Social Issues, 44(3), p. 255+.

[5] Cliggett, L., 2013, “Qualitative Data Archiving in the Digital Age: Strategies for Data Preservation and Sharing," The Qualitative Report, pp. 1-11.

[6] Corti, L., 2007, "Re-Using Archived Qualitative Data - Where, How, Why?,” Archival Science, 7(1), pp. $37-54$.

[7] Kim, Y., and Adler, M., 2015, “Social Scientists' Data Sharing Behaviors: Investigating the Roles of Individual Motivations, Institutional Pressures, and Data Repositories," International Journal of Information Management, 35(4), pp. 408-418.

[8] Burleigh, J. C., and Peterson, H. W., 1967, "Videotapes in Teacher Education," The Elementary School Journal, 68(1), pp. $35-38$. 
[9] Seidel, T., Stürmer, K., Blomberg, G., Kobarg, M., and Schwindt, K., 2011, “Teacher Learning from Analysis of Videotaped Classroom Situations: Does It Make a Difference Whether Teachers Observe Their Own Teaching or That of Others?," Teaching and Teacher Education, 27(2), pp. 259-267.

[10] Carter, D., 2015, “Conceptions of Work and the Materiality of the Classroom,” Newport Beach, CA.

[11] Marsh, B., and Mitchell, N., 2014, "The Role of Video in Teacher Professional Development," Teacher Development, 18(3), pp. 403-417.

[12] Carlson, S., and Anderson, B., 2007, "What Are Data? The Many Kinds of Data and Their Implications for Data ReUse," Journal of Computer-Mediated Communication, 12(2), pp. 635-651.

[13] Gracy, K. F., 2007, “Moving Image Preservation and Cultural Capital,” Library Trends, 56(1), pp. $183-197$.

[14] Harvey, R., 2012, Preserving Digital Materials, de Gruyter Saur, Berlin [u.a.].

[15] Corti, L., 2013, "Infrastructures for Qualitative Data Archiving," Forschungsinfrastrukturen für die qualitative Sozialforschung, Scivero, Berlin, pp. 35-62.

[16] Stigler, J. W., Gallimore, R., and Hiebert, J., 2000, “Using Video Surveys to Compare Classrooms and Teaching Across Cultures: Examples and Lessons From the TIMSS Video Studies,” Educational Psychologist, 35(2), pp. 87-100.

\section{Author Biographies}

Rebecca D. Frank is a Ph.D. Candidate at the University of Michigan School of Information. Her research interests include digital preservation, data reuse, standards and certification for trustworthy digital repositories, risk perception, and disaster planning for digital repositories. Her work has been supported by the National Science Foundation and the Australian Academy of Science.

Kara Suzuka, Ph.D. (Education, Michigan State University) is a research scientist at the University of Michigan School of Education, Teaching \& Learning Exploratory (TLE) where she leads the research and development for the digital repository. Her research involves studies of classroom practice and utilizes multimedia records of classrooms to engage teachers in practice.

Elizabeth Yakel, Ph.D. (Library and Information Science, University of Michigan) is a Professor at the University of Michigan School of Information. Her current research focuses on data reuse in different disciplines and the role of repositories in facilitating reuse. She has been funded by the Institute for Museum and Library Services and the Andrew W. Mellon Foundation, among others. 